\title{
Simple approximation of the singular probability distribution in a nonadiabatically driven system
}

\author{
A. Bandrivskyy, ${ }^{1}$ D. G. Luchinsky, ${ }^{1,2}$ and P. V. E. McClintock ${ }^{1}$ \\ ${ }^{1}$ Department of Physics, Lancaster University, Lancaster LA1 4YB, United Kingdom \\ ${ }^{2}$ Russian Research Institute for Metrological Service, Ozernaya 46, 119361 Moscow, Russia
}

(Received 20 December 2001; revised manuscript received 3 April 2002; published 19 August 2002)

\begin{abstract}
Singular behavior and the formation of plateaus in the probability distribution in a nonadiabatically driven system are investigated numerically in the weak noise limit. A simple extension of the recently introduced logarithmic susceptibility theory is proposed to construct an approximation of the nonequilibrium potential that is valid throughout whole of the phase space.
\end{abstract}

DOI: 10.1103/PhysRevE.66.021108

PACS number(s): 05.40.-a, 02.50.-r, 05.20.-y

The action of an external periodic force on a stochastic system continues to attract intense interest [1-4], first, because it gives rise to important phenomena in a diversity of physical systems including, e.g., lasers [5], proteins [6], and Josephson junctions [7]. Second, even a quite weak periodic excitation can lead to exponentially strong changes in the probability distribution $\rho(x, t)$, thus offering the possibility of using periodic forces to control the system [8-11].

For many years, analytic theory was restricted either to the case of slow driving (adiabatic regime) or to that of fast and weak driving (regime of effective heating by the external force [12]). It was recently proposed that the interesting nonadiabatic regime, lying in between these two extremes, can be treated in terms of a logarithmic susceptibility (LS). This approximation is valid in both the underdamped [9] and overdamped [10] limits. However, up to now, most of the discussion has been restricted to the activation "energy" only, and the range of applicability of the LS approach has not been explored yet.

In this paper we investigate the validity of the LS approximation over the whole of phase space. Analysis of the nonequilibrium potential in the limit of small noise intensities is perfomed using Monte Carlo simulations. The results are compared with numerical integrations of the Fokker-Planck equation (FPE), the LS approximation, and the full Hamiltonian theory of fluctuations. In doing so we show that for a moderate amplitude of the driving force, there is a large region of phase space within which the nonequilibrium potential is almost flat. Within this region, a condition for applicability of the LS does not hold and applying it in the standard way provides a very poor approximation. We propose a simple modification that extends the theory to cover whole of the phase space.

In the limit of small noise intensity $[1,9,10]$ the probability distribution has a WKB [13] form

$$
\rho(x, t)=z(x, t) e^{-S[x, t] / D} .
$$

In the particular case of one-dimensional motion of a particle driven by a periodically modulated field $K(x, t)=-U^{\prime}(x)$ $+F(t)$ and a white Gaussian noise $\xi(t)$,

$$
\dot{x}=K(x, t)+\xi(t), \quad\langle\xi(t)\rangle=0, \quad\langle\xi(t) \xi(0)\rangle=2 D \delta(t),
$$

the action functional

$$
S[x, t]=\frac{1}{4} \int_{-\infty}^{t}[\dot{x}-K(x, t)]^{2} d t
$$

satisfies a variational minimum principle.

The family of trajectories that minimize the action functional (3) covers the coordinate space of the system (2). In general, more than one trajectory arrives to any given point in the coordinate space $[14,15]$. Those providing the global minimum of $S[x, t]$ define the activation "energy" to reach a point $x$ at a moment of time $t$, which plays the role of a nonequilibrium potential $\Phi(x, t)=\min S[x, t][15]$. These trajectories are optimal paths along which the stochastic system fluctuates with overwhelming probability to a given remote state, and they are experimentally observable [16,17]. Thus an analysis of the probability distribution of the nonadiabatically driven system in the limit of small noise intensity requires a solution of the variational problem (3) with subsequent global minimization of the action on the set of all extremal trajectories.

Use of the LS [9,10] substantially simplifies such an analysis. The theory calculates corrections to the activation "energy" induced by the nonadiabatic driving. It does so in terms of the work done by the external field as the system moves along the unperturbed trajectories in thermal equilibrium, expressing the result in terms of a linear susceptibility.

For the archetypal example of the overdamped Duffing oscillator (2) the LS theory provides an analytical solution of the long-standing nonadiabatic escape problem. More recently this theory was extended to find a complete solution of the nonadiabatic escape problem including corrections due to finite noise intensity. The validity of the theory was verified experimentally for escape processes [4]. Encouraging agreement was obtained, which suggested that the LS might be applicable throughout the whole coordinate space of the system (2) in accordance with its original formulation [9].

We now consider fluctuations that are large but not quite sufficient to cause escape from a potential well. In principle, the LS should provide a simple way of estimating their probability distribution, even for nonadiabatic driving. For the sake of definiteness, we consider motion of a Brownian particle in a double-well Duffing potential $U(x)=-x^{2} / 2$ $+x^{4} / 4$ driven by a periodic force $F(t)=A \cos (2 \pi / T) t$, and a zero-mean white Gaussian noise $\xi(t)$. This model has been used for many physical systems (see, e.g., Ref. [18]) includ- 


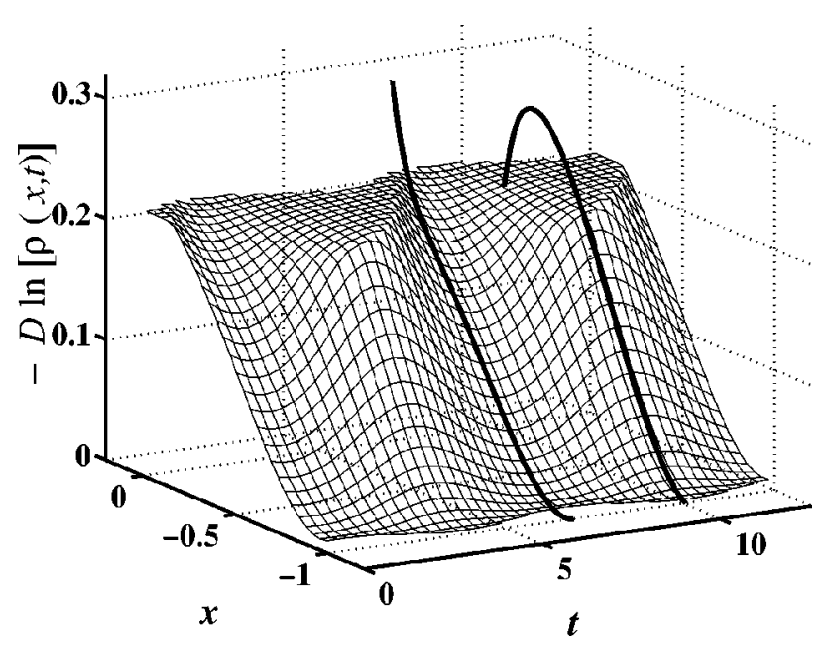

FIG. 1. $-D \ln [\rho(x, t)]$ given by the Monte Carlo simulations in the low noise intensity limit (for $D=0.002$ ). The two bold lines are time sections of $\Phi(x, t)$ predicted by the formula (4). All the figures presented in the paper are calculated for the model system (2) with parameters $T=2 \pi$ and $A=0.1$.

ing those considered recently in the context of stochastic resonance [19].

The nonequilibrium potential of this system is defined as $\Phi(x, t)=\lim _{D \rightarrow 0}-D \ln \rho(x, t)$. It can be measured experimentally in the limit of small noise intensity. The error in such measurements due to the necessarily finite noise intensity $D$ is at least of the order of $D$, so the use of a small noise intensity in such experiments is important. That is why the measurements are usually complicated by the exponentially long observation times needed to build the tails of the distribution. To overcome this problem we use a modified Monte Carlo numerical scheme, of which full details will be presented elsewhere [20]. Here we mention only that the technique has been tested on a number of different systems and that good agrement was obtained with the results obtained using traditional methods for larger noise intensities. The simulation results are compared both with numerical integrations of the Fokker-Planck equation corresponding to Eq. (2) and with the predictions of Hamiltonian fluctuation theory, as will be described below.

An example of $-D \ln [\rho(x, t)]$ obtained by Monte Carlo simulation is shown in Fig. 1. It can be seen from the figure that the $\ln$ of $\rho(x, t)$ approaches a intriguing singular shape with a plateau close to the boundary of the basin of attraction of the quasistable state. It is in good agreement with results from Hamiltonian fluctuation theory and from the numerical solution of the FPE.

To calculate $\ln [\rho(x, t)]$ using the LS theory we will follow the original paper [10]. The basic assumption of the theory is that a moderately strong driving field changes only slightly the unperturbed fluctuational trajectory of the system (2) in thermal equilibrium. If this assumption is valid, the $\Phi(x, t)$ of the nonadiabatically driven overdamped Duffing oscillator can be readily found by substitution into Eq. (3) of the known (see, e.g., Ref. [20]) form of the fluctuational trajectory in thermal equilibrium given by $\dot{x}=U^{\prime}(x)$.

By neglecting the term proportional to $F^{2}(t), \Phi(x, t)$ can be found as a sum of the equilibrium potential $\Delta U(x)$ $=U(x)-U(-1)$ and a correction due to the driving force $[9,10]$

$$
\Phi(x, t)=\Delta U(x)-\int_{-\infty}^{t} \dot{x}_{o}\left(t^{\prime}\right) F\left(t^{\prime}\right) d t^{\prime},
$$

where $\dot{x}_{o}\left(t^{\prime}\right)$ is the velocity of the particle fluctuating along the optimal path in thermal equilibrium. We see that Eq. (4) expresses the corrections to the logarithm of the distribution in terms of usual linear susceptibility. This explains the origin of the term "logarithmic susceptibility." The LS $\chi(t)=$ $-\dot{x}_{o}(i)$. It does not depend on the external force and it is therefore a fundamental property of the unperturbed equilibrium system.

We note that Eq. (4) specifies uniquely the probability distribution for the periodically driven system (2) at every point of the extended coordinate space $(x, t)$. The results of the calculations for two time cross sections are shown in Figs. 1 and 2. It can be seen from the figures that the LS predictions coincide with the results of the Monte Carlo simulations everywhere except in the singular region where there is a plateau close to the boundary of attraction mentioned above. The deviation of the LS predictions from the results of simulations in this region is systematic, much larger than the noise intensity $D$, and clearly requires explanation.

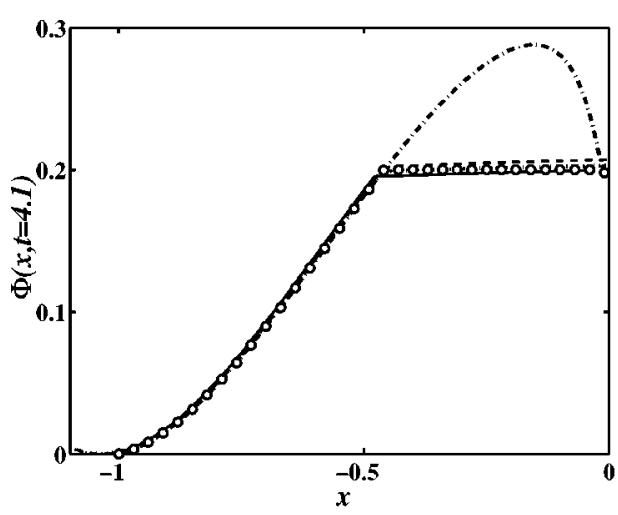

(a)

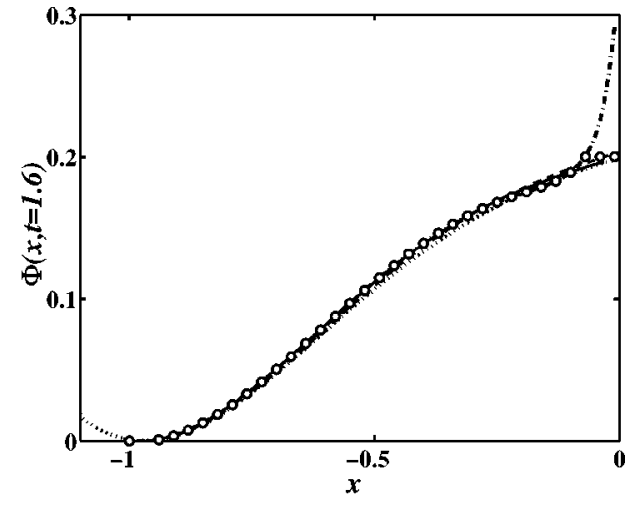

(b)
FIG. 2. Two time sections of $\Phi(x, t)$ for $t=1.6$ and $t=4.1$ given by the unmodified LS (4) (dash-dotted line), the full Hamiltonian theory (solid line), integration of the FPE (for $D=0.002$, dashed line), Monte Carlo simulations (for $D=0.002$, dotted line), and Eq. (4) cutting the action at the level $S_{\text {esc }}$ (circles). 


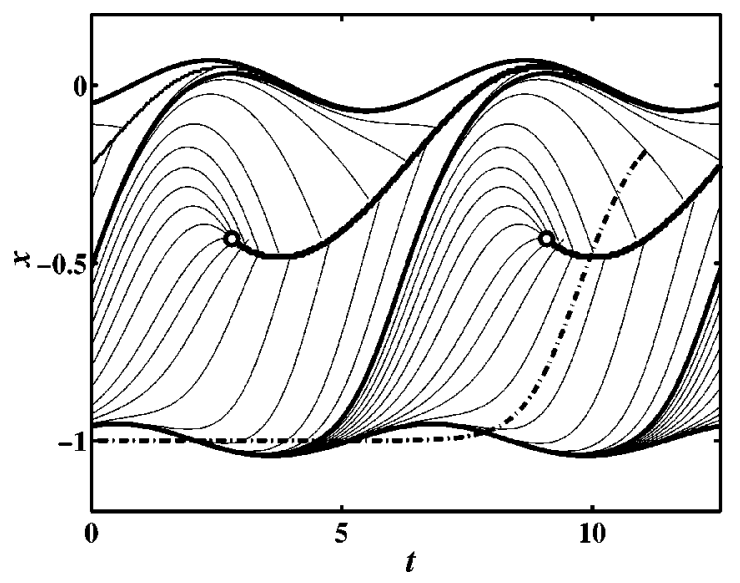

FIG. 3. The pattern of optimal paths. A line that starts at a cusp point (a circle) and approaches unstable periodic orbit is a switching line. Thin solid lines are optimal paths for the nonequilibrium system (2) and dash-dotted line is an optimal path for the corresponding equilibrium system [i.e., for Eq. (2) with $F(t)=0$ ]. Note that equilibrium and nonequilibrium optimal paths that arrive to a singular region of plateau are very different-the original assumption of LS theory breaks down here.

To understand the reason for this disagreement, let us consider the asymptotically $(D \rightarrow 0)$ exact Hamiltonian theory of large fluctuations in a periodically driven system (see Refs. $[21,22])$.

The solution of the variational problem (3) for $S[x, t]$ can be written in terms of the family of extreme trajectories that satisfies the Hamiltonian equations of motion for coordinate $x$ and momentum $p$ of the auxiliary Hamiltonian system $[13,21]$

$$
\begin{gathered}
\frac{d x}{d t}=\frac{\partial H}{\partial p}=K+2 p, \\
\frac{d p}{d t}=-\frac{\partial H}{\partial x}=-p \frac{\partial K}{\partial x} .
\end{gathered}
$$

Along these trajectories the action is given by

$$
\frac{d S}{d t}=p \frac{\partial H}{\partial p}-H=p^{2}
$$

Equations (5) and (6) with appropriate initial conditions give a numerical scheme for computing the family of extreme trajectories and multivalued action manifold $S[x, t]$. Its global minimum is the nonequilibrium potential $\Phi(x, t)[14,15]$.

In Fig. 3, the pattern of optimal paths providing the global minimum to the action functional [22] is shown in the region between $x_{s t}(t)$ and $x_{u}(t) ; x_{s t}(t)$ and $x_{u}(t)$ are the stable and unstable orbits of the system (2), respectively. The bold lines (one per period $T$ ) emanating from $x_{s t}(t)$ and reaching $x_{u}(t)$ asymptotically are the most probable escape paths (MPEPs). These are the paths that provide minimum action for the system to arrive at the boundary of attraction. The singularity in the pattern of optimal paths of the periodically driven Duffing oscillator was investigated earlier [22]. It is deter-

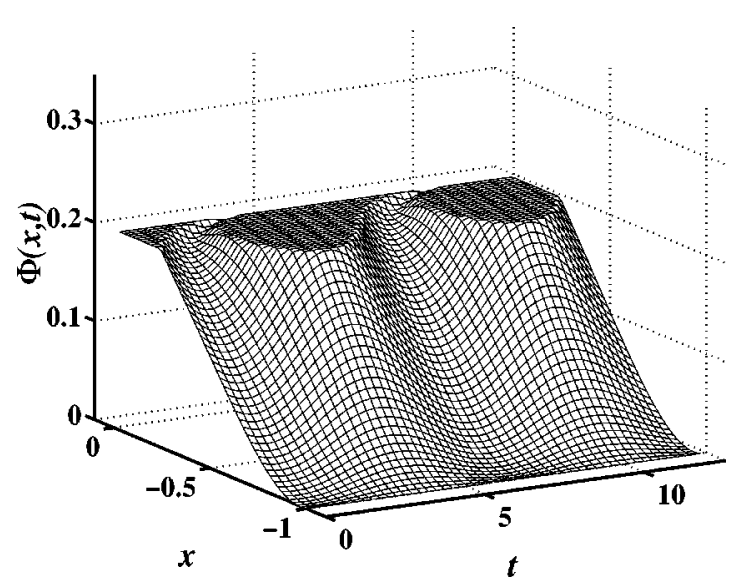

FIG. 4. The cut nonequilibrium potential given by the modified LS approach: a better approximation, cf. Fig. 1.

mined by the topology of the switching line emanating from a cusp point and approaching $x_{u}(t)$ asymptotically. This line separates regions of the coordinate space to which the system arrives by different optimal fluctuation paths.

The region between the switching line and the boundary of attraction $\left[x_{u}(t)\right]$ is a singular plateau with almost constant action. Optimal fluctuation trajectories that arrive in this region first move close to the MPEP and then bend and move away from the boundary of attraction. In this region the basic assumption of the LS theory-that there are only small deviations of the fluctuational paths in the periodically driven system from those in thermal equilibrium-breaks down. As shown in Fig. 3, an optimal equilibrium path (dash-dotted line) arrives to the singular plateau region in a very different way compared to nonequilibrium optimal paths. As a consequence the predictions of the LS theory deviate strongly from the measured nonequilibrium potential in this region.

What can be done to improve the LS approximation in this range? We note, first, that optimal paths reaching the singular region move very close to the MPEP, thus acquiring a value of action that is approximately the same as that acquired along the MPEP ( $S_{\text {esc }}=0.2$ for our choice of parameters). Second, the action acquired along the optimal path anywhere in the region between $x_{s t}(t)$ and $x_{u}(t)$ cannot be larger then the escape "energy" (the activation "energy"). This must mean that the action inside the singular region is almost equal to $S_{\text {esc }}$. We therefore propose that, to improve the LS approximation in this region, we may cut the surface given by Eq. (4) at the level of the escape action $S_{\text {esc }}$.

It can be seen from Figs. 2 and 4 that the predictions of the LS theory, corrected in the singular region, almost coincide with the predictions of the Hamiltonian theory and the results from numerical integration of the FPE over the whole basin of attraction of the stable state, and that they are also in very good agreement with the results of the Monte Carlo simulations [23].

In conclusion, one of the main results of the paper is the observation that the probability distribution may have large, essentially flat regions in phase space. This is a purely dynamical effect that is not associated with the flatness of any potential. Its origin is related to switching between different 
types of optimal fluctuational path, and it is a general feature of optimal paths in periodically driven systems with metastable states. The LS theory, which is a perturbation theory, does not apply within the flat distribution regions. We have shown, however, that the distribution can still be found using a simple and intuitively clear LS-based approach, and we have verified the results through detailed numerical simulations, integration of the Fokker-Planck equation, and solution of the variational problem for large fluctuations. We empha- size that this approach enables one to avoid the necessity of matching two theories in the region of the singularity, and that it can readily be extended to the case of multiharmonic periodic driving.

This work was supported by the Engineering and Physical Sciences Research Council (UK), the Joy Welch Trust (UK), the Russian Foundation for Fundamental Science, and the INTAS.
[1] V.N. Smelyanskiy, M.I. Dykman, and B. Golding, Phys. Rev. Lett. 82, 3193 (1999).

[2] J. Lehmann, P. Reimann, and P. Hänggi, Phys. Rev. Lett. 84, 1639 (2000).

[3] R.S. Maier and D.L. Stein, Phys. Rev. Lett. 86, 3942 (2001).

[4] M.I. Dykman, B. Golding, L.I. McCann, V.N. Smelyanskiy, D.G. Luchinsky, R. Mannella, and P.V.E. McClintock, Chaos 11, 587 (2001).

[5] B.J. Keay, S.J. Allen, J. Galán, J.P. Kaminski, K.L. Campman, A.C. Gosard, U. Bhattacharya, and M.J.W. Rodwell, Phys. Rev. Lett. 75, 4098 (1995).

[6] E.H. Serpersu and T.Y. Tsong, J. Membr. Biol. 74, 191 (1983); J. Biol. Chem. 259, 7155 (1984).

[7] R.L. Kautz, Rep. Prog. Phys. 59, 935 (1996).

[8] F. Julicher, A. Ajdari, and J. Prost, Rev. Mod. Phys. 69, 1269 (1997); C.R. Doering and J.C. Gadoua, Phys. Rev. Lett. 69, 2318 (1992).

[9] M.I. Dykman, H. Rabitz, V.N. Smelyanskiy, and B.E. Vugmeister, Phys. Rev. Lett. 79, 1178 (1997).

[10] V.N. Smelyanskiy, M.I. Dykman, H. Rabitz, and B.E. Vugmeister, Phys. Rev. Lett. 79, 3113 (1997).

[11] B. Golding, L. I. McCann, and M. I. Dykman, in Stochastic and Chaotic Dynamics in the Lakes, edited by D. S. Broomhead, E. A. Luchinskaya, P.V.E. McClintock, and T. Mullin (AIP, Melville, 2000), pp. 34-41.

[12] M.H. Devoret, D. Esteve, J.M. Martinis, A. Cleland, and J. Clarke, Phys. Rev. B 36, 58 (1987); P. Jung, Z. Phys. B: Condens. Matter 76, 521 (1989); B.I. Ivlev and V.I. Melnikov, Phys. Lett. A 116, 427 (1986); A.I. Larkin and Y.N. Ovchinni- kov, J. Low Temp. Phys. 63, 317 (1986); S. Linkwitz and H. Grabert, Phys. Rev. B 44, 11888 (1991); S. Linkwitz and H. Grabert, ibid. 44, 11901 (1991).

[13] M. I. Freidlin and A. D. Wentzel, Random Perturbations in Dynamical Systems (Springer, New York, 1984).

[14] R. Graham and T. Tel, Phys. Rev. Lett. 52, 9 (1984).

[15] R. Graham, in Noise in Nonlinear Dynamical Systems, edited by F. Moss and P. V. E. McClintock (Cambridge University Press, Cambridge, England, 1989), Vol. 1, pp 225-278.

[16] M.I. Dykman, P.V.E. McClintock, V.N. Smelyanski, N.D. Stein, and N.G. Stocks, Phys. Rev. Lett. 68, 2718 (1992).

[17] D.G. Luchinsky and P.V.E. McClintock, Nature (London) 389, 463 (1997).

[18] H. Haken, Rev. Mod. Phys. 47, 67 (1975).

[19] M.I. Dykman, D.G. Luchinsky, R. Mannella, P.V.E. McClintock, N.D. Stein, and N.G. Stocks, Nuovo Cimento D 17, 661 (1995); L. Gammaitoni, P. Hänggi, P. Jung, and F. Marchesoni, Rev. Mod. Phys. 70, 223 (1998); D.G. Luchinsky, R. Mannella, P.V.E. McClintock, and N.G. Stocks, IEEE Trans. Circuits Syst., II: Analog Digital Signal Process. 46, 1205 (1999).

[20] A. Bandrivskyy, D. G. Luchinsky, and P.V.E. McClintock (unpublished).

[21] D. Ludwig, SIAM Rev. 17, 605 (1975).

[22] M.I. Dykman, D.G. Luchinsky, P.V.E. McClintock, and V.N. Smelyanskiy, Phys. Rev. Lett. 77, 5229 (1996).

[23] Note that the slightly higher level of $\Phi(x, t)$ in the singular region given by numerical simulations and integration of FPE is the finite noise intensity effect that vanishes as $D$ approaches the zero limit. 